\title{
Stress rapidly dysregulates the glutamatergic synapse in the prefrontal cortex of cocaine-withdrawn adolescent rats
}

\author{
Lucia Caffino ${ }^{1,2}$, Francesca Calabrese ${ }^{1,2}$, Giuseppe Giannotti ${ }^{1,2}$, Alessandro Barbon ${ }^{3}$, \\ Michel M. M. Verheij ${ }^{4}$, Giorgio Racagni ${ }^{1,2,5}$ \& Fabio Fumagalli',
}

Centro di Neurofarmacologia, Dipartimento di Scienze Farmacologiche e Biomolecolari, Università degli Studi di Milano, Milan, Italy', Collaborative Center of Department of Antidrug Policies, Presidency of the Council of Ministers, Rome, Italy², Division of Biology and Genetics, Department of Molecular and Translational Medicine, National Institute of Neuroscience, University of Brescia, Brescia, Italy ${ }^{3}$, Department of Cognitive Neuroscience, Donders Institute for Brain, Cognition and Behaviour, Radboud University Nijmegen Medical Centre, Nijmegen, The Netherlands ${ }^{4}$ and I.R.C.C.S. San Giovanni di Dio-Fatebenefratelli, Brescia, Italy

\begin{abstract}
Although several lines of evidence have shown that chronic cocaine use is associated with stress system dysregulation, the underlying neurochemical mechanisms are still elusive. To investigate whether the rapid stress-induced response of the glutamatergic synapse was influenced by a previous history of cocaine, rats were exposed to repeated cocaine injections during adolescence [from postnatal day (PND) 28-42], subjected to a single swim stress (5 minutes) three days later (PND 45) and sacrificed 15 minutes after the end of this stressor. Critical determinants of glutamatergic homeostasis were measured in the medial prefrontal cortex $(\mathrm{mPFC})$ whereas circulating corticosterone levels were measured in the plasma.

Exposure to stress in saline-treated animals did not show changes in the crucial determinants of the glutamatergic synapse. Conversely, in cocaine-treated animals, stress dynamically altered the glutamatergic synapse by: (1) enhancing the presynaptic vesicular mediators of glutamate release; (2) reducing the transporters responsible for glutamate clearance; (3) increasing the postsynaptic responsiveness of the N-methyl-D-aspartate subunit GluN1; and (4) causing hyperresponsive spines as evidenced by increased activation of the postsynaptic cdc42-Pak pathway.

These findings indicate that exposure to cocaine during adolescence sensitizes mPFC glutamatergic synapses to stress. It is suggested that changes in glutamatergic signaling may contribute to the increased sensitivity to stress observed in cocaine users. Moreover, glutamatergic processes may play an important role in stress-induced reinstatement of cocaine seeking.
\end{abstract}

Keywords: Adolescence, cocaine, glia, glutamate, prefrontal cortex, stress.

Correspondence to: Fabio Fumagalli, Centro di Neurofarmacologia, Dipartimento di Scienze Farmacologiche e Biomolecolari, Università degli Studi di Milano, Via Balzaretti 9, 20133 Milano, Italy. E-mail: Fabio.Fumagalli@unimi.it

\section{INTRODUCTION}

Drug addiction is a chronic relapsing disorder hypothesized to be produced by drug-induced neuroplasticity that renders individuals vulnerable to craving-inducing stimuli. Besides alterations in central dopamine homeostasis (Berridge \& Robinson 1998), addiction liability has also been associated to changes in prefrontal-accumbens glutamate transmission (Kalivas, Volkow \& Seamans 2005; Gipson, Kupchik \& Kalivas 2013). Several studies have investigated the role of glutamate in the nucleus accumbens (Huang et al. 2011; Xie et al. 2012; Purgianto et al. 2013) while we know less about cocaineinduced glutamate plasticity in the medial prefrontal cortex (mPFC), although the mPFC has been described as a key site for compulsive drug-seeking (Everitt \& Robbins 2005). In fact, long-term exposure to cocaine leads to contrasting results with respect to glutamate receptor modulation (Hemby, Horman \& Tang 2005; Freeman et al. 2008; Kasanetz et al. 2013) whereas withdrawal

The authors declare no conflict of interest in relation to the work herein described. 
from cocaine leads to robust changes in the redistribution of these cortical receptors (Ghasemzadeh, Mueller \& Vasudevan 2009; Ben-Shahar et al. 2013).

Among the factors that cause relapse to drug-seeking, stress plays an important role. In fact, cocaine users are extremely sensitive to stressful events (Sinha et al. 2003; Fox et al. 2008). Studies in animals have shown that acute stress can induce relapse to drug seeking following chronic exposure to cocaine (Erb, Shaham \& Stewart 1996; Ahmed \& Koob 2005), but the contribution of glutamate to stress-induced cocaine reinstatement is still elusive. In the current study, we incorporated a paradigm of acute swim stress to investigate, for the first time in detail, the functional responsiveness of the mPFC glutamate system in animals with a prior history of cocaine. We have used adolescent rats because these animals are more vulnerable to cocaine when compared with adult rats (Wong et al. 2013).

Glutamate is the major excitatory neurotransmitter in the brain. It is stored within intracellular secretory vesicles via the action of vesicular glutamate transporters (vGLUTs) that do not only serve storage functions, but are also involved in the regulation of glutamate release (Bellocchio et al. 2000). Once released, glutamate activates postsynaptic ionotropic and metabotropic receptors before removal from the extracellular space into glial cells through the action of excitatory amino acid transporters (EAAT1 and EAAT2; Bridges et al. 2012). Once inside the glial cells, glutamine synthetase (GS) converts glutamate into glutamine, which is then released by glial cells and taken up by glutamate neurons (Broer \& Brookes 2001) thereby replenishing the presynaptic vesicular stores of glutamate. In addition, glutamate homeostasis is modulated also by an antiporter that exchanges extracellular cystine for intracellular glutamate and is also responsible of the extra-synaptic release of glutamate (Bridges et al. 2012).

We show that swim stress rapidly dysregulates the expression of the components of the glutamatergic synapse in the mPFC of cocaine-withdrawn adolescent rats mentioned earlier. The observed cocaine-induced sensitization of the glutamatergic synapse to stress may contribute to the increased sensitivity to stress observed in cocaine users as well as to stress-induced reinstatement of cocaine seeking.

\section{MATERIAL AND METHODS}

\section{Experimental procedures}

The adolescent rats used in this study were generated by mating Sprague Dawley rats weighting $250 \mathrm{~g}$ (Charles River, Calco, Italy) and housed under standard conditions of temperature and humidity under artificial light (from
7:00 AM to 7:00 PM). A maximum of two male siblings was taken from each litter in order to reduce 'litter effects' (Chapman \& Stern 1978). Male rats were treated subcutaneously with cocaine $(20 \mathrm{mg} / \mathrm{kg} /$ day; MacFarlanSmith, Edinburgh, UK) or saline from postnatal day 28 (PND 28) to PND 42, a period that roughly approximates adolescence in humans (Collins \& Izenwasser 2004).

Following the end of this treatment, animals were left undisturbed in their home cages until PND 45 when they were randomly divided into four experimental groups. Group 1 was formed by rats exposed to saline from PND 28 to 42 and sacrificed on PND 45 without any further manipulation; group 2 was formed by rats exposed to saline from PND 28 to 42 and sacrificed on PND 45 following acute stress; group 3 was formed by rats exposed to cocaine from PND 28 to 42 and sacrificed on PND 45 without any further manipulation; group 4 was formed by rats exposed to cocaine from PND 28 to 42 and sacrificed on PND 45 following acute stress. A schematic picture is shown in Fig. 1a. Swim stress was chosen since we have previously shown that it rapidly modulates the phosphorylation of glutamate postsynaptic receptors in the brain of adult rats, a measure of activation of the glutamate synapse (Fumagalli et al. 2009b). Also, this stress allows us to have behavioral measures of prodepressive symptoms, which are known to occur during the initial phase of cocaine withdrawal in humans and animal models (Gawin 1991; Perrine et al. 2008). Rats were individually placed in glass cylinders containing water $\left(24^{\circ} \mathrm{C}\right)$ at a depth of $30 \mathrm{~cm}$. Depth of the water was adequate to prevent rats from touching the bottom of the cylinder with their tails. Water was replaced between each test and temperature was monitored constantly. Three independent investigators, blind to the experimental design, measured the time the animals were immobile during the 5 minutes test. Immobility was defined as lack of movement of three paws and only minimal movement of the fourth or lack of movement of all four paws.

Animals were sacrificed 15 minutes after the end of the swim stress. Following the sacrifice, the $\mathrm{mPFC}$ (defined as $\mathrm{Cg} 1, \mathrm{Cg} 3$ and IL subregions) corresponding to plates 5-9 of the atlas of Paxinos and Watson (Paxinos and Watson, 2005) has been immediately dissected from 2-mm thick slices, frozen on dry ice and stored at $-80^{\circ} \mathrm{C}$. Trunk blood from each rat was collected in heparinized tubes for quantification of corticosterone plasma levels. Procedures involving animals and their care were conducted in conformity with the institutional guidelines that are in compliance with national (D.L. n. 116, G.U., supplement 40, 18 Febbraio, 1992, Circolare no. 8, G.U., 14 Luglio, 1994) and international laws and policies (EEC Council Directive 86/609, OJL 358, 1, December 12, 1987; Guide for the Care \& Use of Laboratory Animals, U.S. National Research Council, 1996). 
(a)

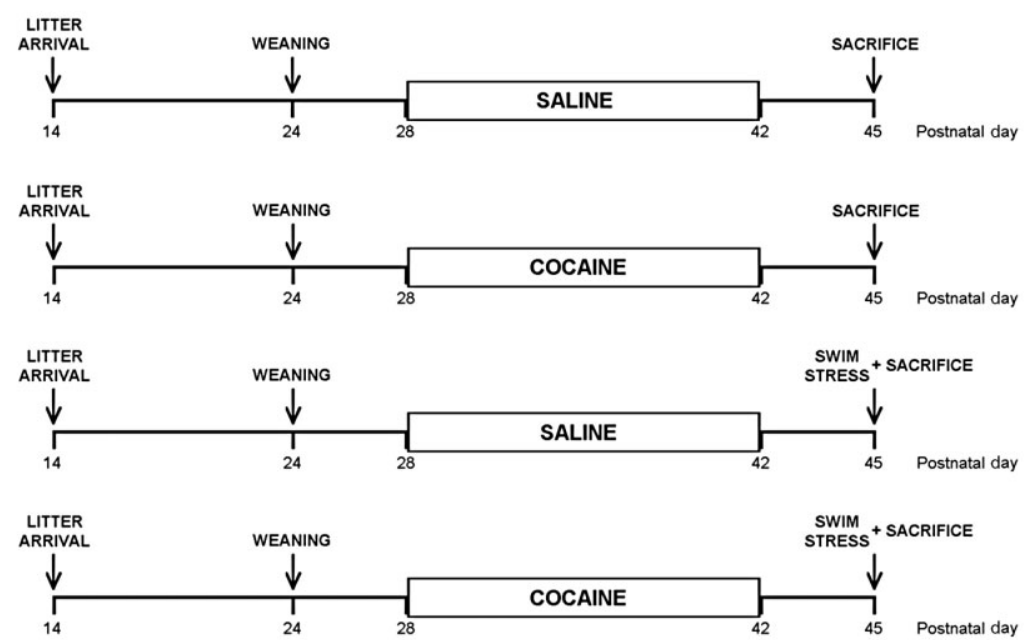

(b)

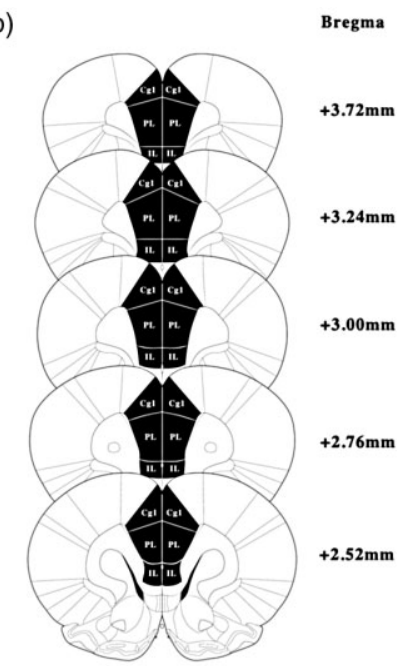

(c)

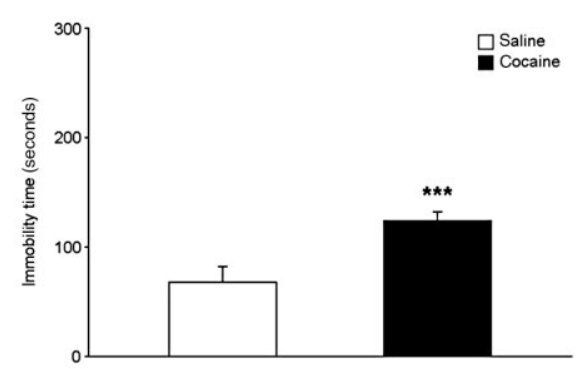

(d)

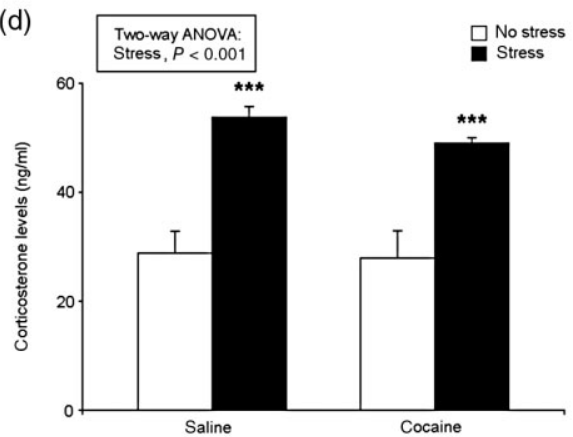

Figure I Schematic representation of the experimental procedure, specific coordinates of medial prefrontal cortex dissection and general analyses characterizing the stress experiment.

Panel (a) shows the experimental paradigm. Animals were treated with cocaine $(20 \mathrm{mg} / \mathrm{kg})$ or saline during early adolescence [postnatal day (PND) 28-PND 42]. After the end of the adolescent treatment, animals were left undisturbed in their home cages. On PND 45, half of the animals exposed to cocaine or to saline, were subjected to 5 minutes of swim stress and sacrificed 15 minutes after the end of this stressor. The other half of the animals was left in their home cages and sacrificed at the same time as their swim stress-exposed counterparts. Panel (b) shows the dissection of the medial prefrontal cortex. This procedure was undertaken according to the coordinates indicated by the atlas of Paxinos and Watson, 2005 (please see the Material and Methods section). The medial prefrontal cortex was then stored at $-80^{\circ} \mathrm{C}$ until processing.

Panel (c) shows the time (seconds) the animals spent immobile during the 5 minutes of swim stress. Measurements were taken by three independent investigators who were blind to the experimental design ( $* * * *<0.00$ I, unpaired Student's $t$-test).

Panel (d) shows the levels of circulating corticosterone (expressed in $\mathrm{ng} / \mathrm{ml})$. The main effects of analysis of variance appear at the top of the panel $(* * * P<0.001)$

RNA preparation and gene expression analysis by quantitative real-time PCR

RNA measures were taken in the same animals as the protein measures. Total RNA was isolated by single step guanidinium isothiocyanate/phenol extraction using PureZol RNA isolation reagent (Bio-Rad Laboratories, Segrate, Milan, Italy) according to the manufacturer's instructions and quantified by spectrophotometric analysis (Caffino, Racagni \& Fumagalli 2011). Following total RNA extraction, the samples were processed for realtime reverse transcription polymerase chain reaction (real-time RT-PCR) to assess mRNA levels, as previously described (Fumagalli et al. 2012). Briefly, an aliquot of each sample was treated with DNase to avoid DNA contamination. RNA was analyzed by TaqMan qRTPCR instrument (CFX384 real time system, Bio-Rad Laboratories) using the iScript ${ }^{\mathrm{TM}}$ one-step RT-PCR kit for probes (Bio-Rad Laboratories). Each experimental group was composed of at least six rats. Samples were run in 384 well formats in triplicate as multiplexed reactions. Data were analyzed with the comparative threshold cycle $(\Delta \Delta \mathrm{Ct})$ method using $36 \mathrm{~B} 4, \beta$-actin and $18 S$ as reference genes (Barbon et al. 2010). The primer efficiencies were experimentally set up for each couple of primers. 
Probes and primers were purchased from Eurofins MWG-Operon. Their sequences are shown below. Thermal cycling was initiated with an incubation at $50^{\circ} \mathrm{C}$ for 10 minutes (RNA retrotranscription) and then at $95^{\circ} \mathrm{C}$ for 5 minutes (TaqMan polymerase activation). After this initial step, 39 cycles of PCR were performed. Each PCR cycle consisted of heating the samples at $95^{\circ} \mathrm{C}$ for 10 seconds to enable the melting process and then for 30 seconds at $60^{\circ} \mathrm{C}$ for the annealing and extension reaction.

The following is the list of the primers and probe used in our experiments:

- vGLUT1: Forward primer 5'-ACTGCCTCACCTTGTC ATG-3', Reverse Primer 5'-GTAGCTTCCATCCCGAA ACC-3', Probe 5'-CTTTCGCACATTGGTCGTGGACAT $\mathrm{T}-3^{\prime}$;

- vGAT: Forward primer 5'-ACGACAAACCCAAGAT CACG-3', Reverse Primer 5'-GTAGACCCAGCACGAA CATG-3', Probe 5'-TTCCAGCCCGCTTCCCACG-3';

- GAD67: Forward primer 5'-ATACTTGGTGTGGCGT AGC-3', Reverse Primer 5'-AGgAAAGCAGGTTCTT GGAG-3', Probe 5'-AAAACTGGGCCTGAAGATCTGT GGT-3';

- EAAT1: Forward primer 5'-GGGTTTTCATTGGAGGG TTG-3', Reverse Primer 5'-ACGGGAAGCACAAATC TGG-3', Probe 5'-TGATGGGCAGGGTGGCAGAA-3';

- EAAT2: Forward primer 5'-TTGCTGGCATTTTCCAA GC-3', Reverse Primer 5'-TTAATGGTTGCTCCGACTG G-3', Probe 5'-CAAGCGTGTGACCAGATTCGTCCT-3';

- $\mathrm{X}_{\mathrm{C}}^{-}$: Forward primer 5'-TCTTCGATACAAACGCCCAG3', Reverse Primer 5'-CGAGTAAAGGGAGAGGACA AC-3', Probe 5'-CCATGAAGAGGCAGGTGAAGGAG AA-3';

- GS: Forward primer 5'-ATGGTCTGAGGTGCATTGAG3', Reverse Primer 5'-TGATGTTGGAGGTTTCGTGG3', Probe 5'-ATGTGGTACTGGTGCCTCTTGCTC-3';

- Cdc42: Forward primer 5'-AAGGCTGTCAAGTATGT GGAG-3', Reverse Primer 5'-GCTCTGGAGATGCGTTC ATAG-3', Probe 5'-CCTGCGGCTCTTCTTCGGTTCT-3';

- $\beta$-actin: Forward primer 5'-CACTTTCTACAATGAG CTGCG-3', Reverse Primer 5'-CTGGATGGCTACGTA CATGG-3', Probe 5'-TCTGGGTCATCTTTTCACGGTT GGC-3';

- 18S: Forward primer 5'-GTAACCCGTTGAACCCCA TT-3', Reverse Primer 5'-CCATCCAATCGGTAGTAGC G-3', Probe 5'-TGCAATTATTCCCCATGAACGAGG-3';

- 36B4: Forward primer 5'-TTCCCACTGGCTGAAAAG GT-3', Reverse Primer 5'-CGCAGCCGCAAATGC-3', Probe 5'-AAGGCCTTCCTGGCCGATCCATC-3'.

\section{Preparation of protein extracts and Western blot analyses}

mPFC was homogenized in a glass-glass potter using a cold buffer containing $0.32 \mathrm{M}$ sucrose, $1 \mathrm{mM}$
$\mathrm{N}$-2-hydroxyethylpiperazine- $\mathrm{N}^{\prime}$-2-ethanesulfonic acid (HEPES) solution, $0.1 \mathrm{mM}$ ethylene glycol tetraacetic acid (EGTA), $0.1 \mathrm{mM}$ phenylmethanesulfonyl fluoride, $\mathrm{pH}=7.4$, in presence of a complete set of protease inhibitors and a phosphatase inhibitor cocktail. Crude synaptosomal fraction was prepared as previously described (Fumagalli et al. 2009a). The homogenized tissues were centrifuged at $1000 \mathrm{~g}$ for 10 minutes; the resulting supernatant was centrifuged at $9000 \mathrm{~g}$ for 15 minutes to obtain the pellet corresponding to the crude synaptosomal fraction, which was resuspended in a buffer containing $20 \mathrm{mM}$ HEPES, $0.1 \mathrm{mM}$ dithiothreitol, 0.1 mM EGTA, in presence of a complete set of protease inhibitors and a phosphatase inhibitor cocktail. Total proteins have been measured in the crude synaptosomal fraction by the Bio-Rad Protein Assay (Bio-Rad Laboratories). Ten micrograms of proteins for each sample were run on an sodium dodecyl sulfate- $10 \%$ polyacrylamide gel under reducing conditions and then electrophoretically transferred onto nitrocellulose membranes (GE Healthcare, Milan, Italy). Blots were blocked 1 hour at room temperature with $10 \%$ non-fat dry milk in tris buffered saline $+0.1 \%$ Tween-20 buffer, incubated with antibodies against the phosphorylated forms of the proteins and then stripped and reprobed with the antibodies against corresponding total proteins.

The conditions of the primary antibodies were the following: anti phospho-GluN1 (Ser896; 1:1000, Affinity Bioreagents, Golden, CO, USA), anti phospho$\alpha$ CaMKII (T286; 1:2000, Thermo Scientific, Waltham, MA, USA), anti phospho-GluN2B (Ser1303; 1:1000, Upstate, Lake Placid, NY, USA), anti phospho-GluA1 (Ser831; 1:500, Affinity Bioreagents), anti phosphoGluA2 (Ser880; 1:1000, Abcam, Cambridge, UK), anti phospho-Pak1 (Thr423; 1:1000, Cell Signaling Technology Inc., Beverly, MA, USA), anti total GluN1 (1:1000, Zymed Laboratories, San Francisco, CA, USA), anti total $\alpha$ CaMKII (1:5000, Chemicon, Temecula, CA, USA), anti total GluN2B (1:1000, Santa Cruz Biotechonology, Santa Cruz, CA, USA), anti total GluN2A (1:1000, Zymed Laboratories), anti total GluA1 (1:2000, Upstate), anti total GluA2 (1:2000, Alomone, Jerusalem, Israel), anti total Pak1 (1:1000, Cell Signaling), anti-Arc (activity-regulated cytoskeletalassociated protein; 1:500, BD Transduction Laboratories, San Jose, CA, USA) and anti $\beta$-actin (1:10 000, Sigma-Aldrich). Immunocomplexes were visualized by chemiluminescence using the Chemidoc MP Imaging System (Bio-Rad Laboratories). The activation of the proteins investigated were expressed as a ratio between the phosphorylated and the respective total forms and analyzed using the Image Lab software from Bio-Rad Laboratories. 


\section{Analysis of plasma corticosterone levels}

Plasma was separated by centrifugation $(6500 \mathrm{~g}$ for 10 minutes) and corticosterone levels were determined by an enzyme-linked immunosorbent assay using a commercial kit, according to the manufacturers' instructions (IBL, Hamburg, Germany).

\section{STATISTICAL ANALYSIS}

Data were collected in individual animals (independent determinations) and are presented as means and standard errors. Changes produced by cocaine treatment and acute stress alone as well as by their combination were analyzed using a two-way analysis of variance (ANOVA), with adolescent cocaine treatment and acute stress as independent variables. When dictated by relevant interaction terms, single contrast post-hoc test (SCPHT) was used to characterize differences among individual groups of rats. However, when no interaction between cocaine treatment and stress was observed, only the main effects were reported. The immobility time, measured during the swim stress, was analyzed by an unpaired Student's $t$ test. Statistical significance was assumed at $P<0.05$.

\section{RESULTS}

Figure 1a illustrates the experimental paradigm that was designed to investigate whether the rapid stress-induced response of the glutamatergic synapse was influenced by a previous history of cocaine. mPFC was dissected as depicted in Fig. 1b. Figure 1c shows the behavioral response to the 5 minutes stress in both saline- and cocaine-treated rats. We measured the time that the animals were immobile during the 5 minutes of forced swimming, an index of pro-depressive symptoms that are known to be related, at least in part, to increased glutamate transmission (Sanacora, Treccani \& Popoli 2012). Interestingly, cocaine-treated rats showed higher immobility when compared with saline-treated rats $(+81 \%, P<0.01$, Student's $t$-test; Fig. $1 \mathrm{c})$. We also measured the plasma levels of corticosterone and found a significant effect of stress $\left(F_{1,33}=57.74 ; P=0.22 \mathrm{E}-07\right.$; two-way ANOVA), but no cocaine $\times$ stress interaction $\left(F_{1,33}=0.86 ; \quad P=0.358 ;\right.$ two-way ANOVA $)$. Notably, cocaine alone did not alter the plasma levels of corticosterone (Fig. 1d).

We next measured the expression of several molecular determinants of the glutamate synapse in response to the combination of adolescent exposure to cocaine and acute stress. Given that the short interval between the end of stress and sacrifice ( 15 minutes) is likely to favor changes that occur rapidly, we focused on measuring gene expression and/or phosphorylation of the different glutamate determinants, since these represent the initial responses, whereas changes in corresponding protein levels take much longer.

Figure 2 shows the contribution of cortical glial cells to the effects produced by the combination of cocaine and stress. Considering the glutamate transporters EAAT1 and EAAT2, their response to stress was influenced by prior cocaine history. Two-way ANOVA showed a cocaine $\times$ stress interaction for EAAT1 $\left(F_{1,35}=5.48\right.$; $P=0.026)$ and EAAT2 $\left(F_{1,30}=6.77 ; P=0.015\right.$; Fig. $2 \mathrm{a}$ $\& 2 b)$. The single intergroup comparisons revealed that stress decreased the levels of these transporters in cocaine-treated animals (EAAT1: $-14 \%$ versus cocaine-no stress, $F_{1,17}=6.15, P=0.038$; EAAT2: $-23 \%$ versus cocaine-no stress, $F_{1,15}=12.07, \quad P=0.0036$; SCPHT), but not in saline-treated rats (EAAT1: $+4 \%$ versus saline-no stress, $F_{1,18}=0.65, P=0.854$; EAAT2: $+2 \%$ versus saline-no stress, $F_{1,15}=0.06, P=1.632$; SCPHT; Fig. 2a \& 2b). We also measured the expression of another transporter expressed by glial cells, such as the glucose transporter 1 (Glut-1). No changes in Glut-1 mRNA levels were found suggesting that the combination of cocaine and stress selectively targets glial glutamate transport (data not shown).

Besides glutamate transporters, extracellular glutamate levels may also be modulated via the regulation of the glial cystine/glutamate antiporter (system $X_{\mathrm{c}}^{-}$) that exchanges extracellular cystine for intracellular glutamate (Bridges et al. 2012). Two-way ANOVA indicated cocaine $\times$ stress interaction $\left(F_{1,33}=6.78\right.$; $P=0.015$ ) for system $X_{c}^{-}$mRNA levels (Fig. 2c). The analysis of the single treatment effects revealed a significant reduction of system $X_{\mathrm{c}}^{-}$in the $\mathrm{mPFC}$ of animals exposed to cocaine and stress $(-19 \%$ versus cocaine-no stress, $F_{1,17}=6.16, P=0.038$; SCPHT) with no effects in saline-treated rats $(+9 \%$ versus saline-no stress, $F_{1,16}=1.42, \quad P=0.486$; SCPHT; Fig. 2c). To further characterize the contribution of glial cells, we analyzed the effect of the combination of cocaine and stress on the enzyme that converts glutamate into glutamine in the glia, i.e. GS. Two-way ANOVA indicated cocaine $\times$ stress interaction $\left(F_{1,33}=5.71 ; P=0.024\right)$ for GS mRNA levels (Fig. 2d). We subdivided the data for individual intergroup comparisons and found that stress evoked a significant elevation of GS mRNA levels only in rats that had received cocaine during adolescence $(+26 \%$ versus cocaine-no stress, $F_{1,16}=16.61, P=0.0006$; SCPHT) with no effects in saline-treated rats $(+7 \%$ versus saline-no stress; $F_{1,17}=0.62, P=0.878$; SCPHT; Fig. $2 d$ ).

Next we investigated the presynaptic terminal (Fig. 3). Two way ANOVA indicated a cocaine $\times$ stress interaction $\left(F_{1,30}=27.78 ; P=0.000016\right)$ for vGLUT1 mRNA levels (Fig. 3a). Thus, we subdivided the data for individual intergroup comparisons. Stress evoked a marked 
(a)

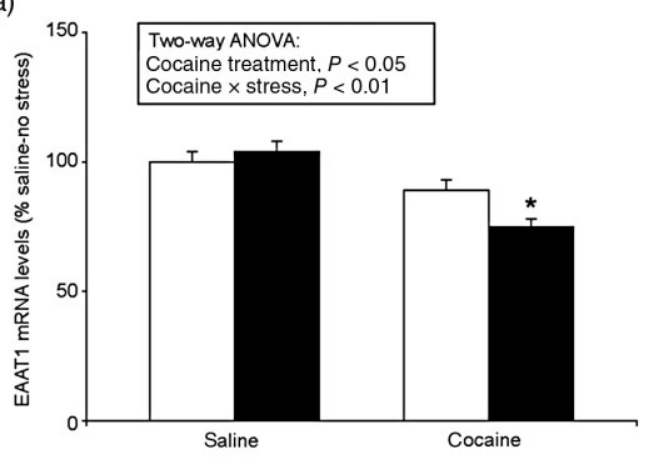

(c)

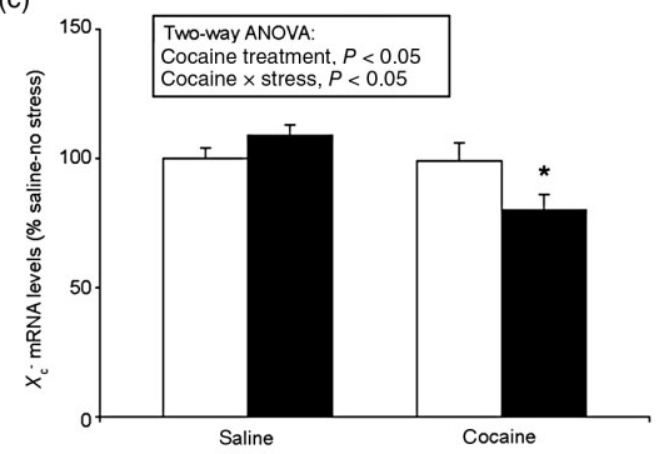

(b)

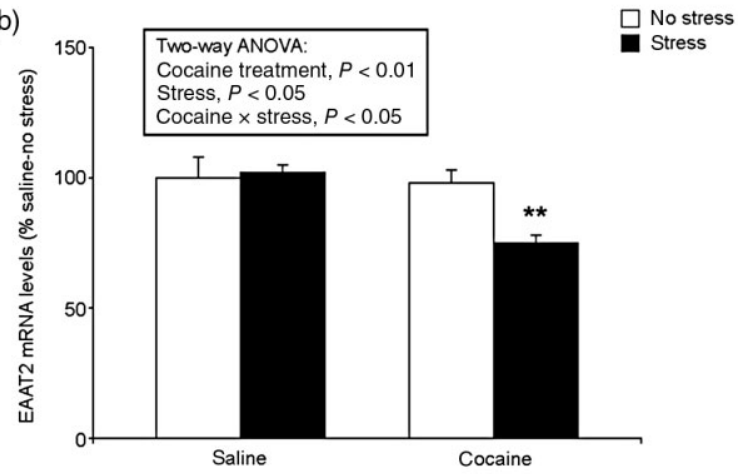

(d)

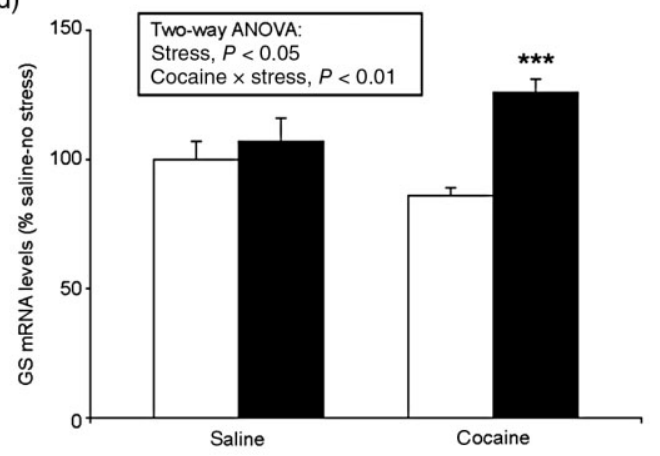

Figure 2 Effect of repeated cocaine treatment during adolescence [postnatal day (PND) 28 to PND 42] on glial determinants of glutamate neurotransmission and on the subsequent response to acute stress assessed at PND 45 in the medial prefrontal cortex.

Panel (a) and (b) show the glial glutamate transporter mRNA levels, EAATI and EAAT2; panel C shows the glial glutamate exchanger mRNA levels, $X_{c}^{-}$, while panel $D$ shows the glutamine synthetase mRNA levels, GS. Asterisks denote the significant effect of the acute stress in cocaine-treated rats versus cocaine-no stress $(* P<0.05$; $* * P<0.0$ I and $* * * P<0.00$ I)

up-regulation of vGLUT1 mRNA levels only in rats that had received cocaine during adolescence $(+106 \%$ versus cocaine-no stress, $F_{1,12}=36.08, P=0.000004$; SCPHT) with no effects in saline-treated rats $(-14 \%$ versus saline-no stress, $F_{1,18}=0.95, P=0.676$; SCPHT; Fig. 3a). The analysis of the vesicular gamma-aminobutyric acid (GABA) transporter (vGAT) mRNA levels revealed only a main effect of cocaine treatment $\left(F_{1,33}=22.07\right.$; $P=0.00006$; two-way ANOVA; Fig. 3b) with no significant interaction between cocaine and stress $\left(F_{1,33}=0.125 ; P=0.727\right.$; two-way ANOVA; Fig. 3b). We next analyzed GAD67 mRNA levels, the GABA synthesizing enzyme isoform highly expressed in the central nervous system (Soghomonian \& Martin 1998). Twoway ANOVA indicated a significant cocaine $\times$ stress interaction $\left(F_{1,32}=4.54 ; P=0.041\right)$. The analysis of the single treatment effects revealed a significant reduction of GAD67 in the mPFC of animals exposed to cocaine and stress $\left(-28 \%\right.$ versus cocaine-no stress, $F_{1,15}=12.74$, $P=0.003$; SCPHT) with no effects in saline-treated rats $\left(-5 \%\right.$ versus saline-no stress, $F_{1,17}=0.472, P=0.996$; SCPHT; Fig. 3c).

Next, we investigated the postsynaptic terminal and analyzed the activation of the obligatory N-methyl-D- aspartate (NMDA) receptor subunit, GluN1, in the crude synaptosomal fraction, expressed as a ratio between the phosphorylated and the total levels of protein. As shown in Fig. 4a, two-way ANOVA indicated a significant cocaine $\times$ stress interaction $\left(F_{1,22}=5.46 ; P=0.031\right)$. Examining the individual treatment effects, we found that swim stress enhanced the phosphorylation levels of GluN1 in cocainetreated rats $\left(+66 \%\right.$ versus cocaine-no stress; $F_{1,11}=$ 18.60, $P=0.0008$; SCPHT) with no effects in salinetreated rats $\left(+15 \%\right.$ versus saline-no stress, $F_{1,11}=1.02$, $P=0.652$; SCPHT; Fig. 4a). No changes in the total levels of NMDA GluN1 were found (data not shown). We also measured the phosphorylation and total levels of the other NMDA (GluN2A, GluN2B) as well as AMPA receptor subunits (GluA1 and GluA2) and found no significant effects in any of these receptors (Table 1), suggesting that the combination of cocaine and stress specifically activates the obligatory NMDA receptor subunit, GluN1.

Given that the acute activation of the NMDA receptor may lead to long-term structural and functional changes in spine plasticity through the modulation of CaMKIICdc42-Pak1 transduction pathway (Murakoshi, Wang \& Yasuda 2011), we also investigated these crucial effectors of such action. As shown in Fig. 4b, two-way ANOVA 
(a)

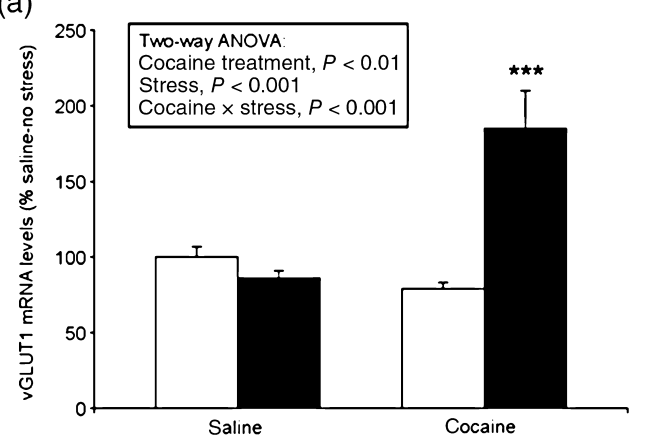

(b)

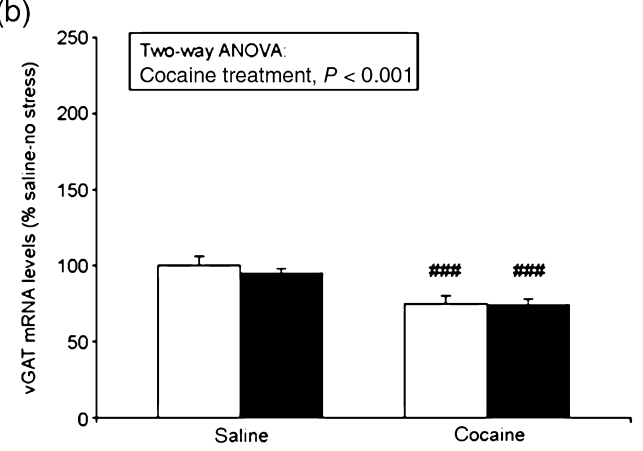

(c)

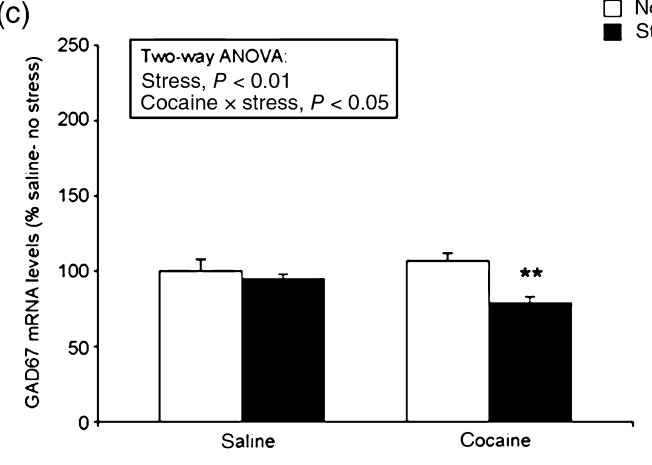

Figure 3 Effect of repeated cocaine treatment during adolescence (PND28 to PND 42) on presynaptic determinants of glutamate transmission.

Panel (a) shows the vesicular glutamate transporter ( $V G L U T I$ ) mRNA levels; panel (b) shows the vesicular gamma-aminobutyric acid transporter (VGAT) mRNA levels; panel (c) shows glutamate decarboxylase 67 (GAD 67) mRNA levels. The main effects of analysis of variance appear at the top of the panel $\left(\#^{\#} P<0.00\right.$ I). Asterisks denote the significant effect of the acute stress in cocaine-treated rats versus cocaine-no stress $(* * P<0.01$; **** $P<0.00$ I)

indicated a significant stress effect on the activation of $\alpha$ CaMKII $\left(F_{1,19}=10.52 ; P=0.005\right)$. Post hoc testing indicated that acute stress enhanced the phosphorylation of $\alpha$ CaMKII in cocaine-withdrawn rats $(+73 \%$ versus cocaine-no stress, $F_{1,10}=6.38, P=0.044$; SCPHT), but not in saline-treated rats $(+40 \%$ versus saline-no stress, $F_{1,9}=4.23, P=0.112$; SCPHT; Fig. 4b).
Two-way ANOVA indicated a cocaine $\times$ stress interaction $\left(F_{1,35}=27.61 ; P=0.00001\right)$ for $\mathrm{Cdc} 42 \mathrm{mRNA}$ levels. Examining the individual treatment effects, we found that acute stress caused a significant elevation of Cdc42 mRNA levels in the mPFC of cocaine-treated rats $\left(+43 \%\right.$ versus cocaine-no stress, $F_{1,17}=31.12$, $P=0.000$ 004; SCPHT), but not in saline-treated rats $\left(-14 \%\right.$ versus cocaine-no stress, $F_{1,18}=27.61, \quad P=$ 0.164; SCPHT; Fig. 4c). The analysis of the activation of Pak1 kinase (expressed as ratio between the phosphorylated and total levels of the protein) also revealed a significant interaction between cocaine and stress $\left(F_{1,20}=5.44 ; P=0.033\right.$; two-way ANOVA $)$ with stress increasing Pak1 phosphorylation levels in cocainetreated rats $\left(+49 \%\right.$ versus cocaine-no stress, $F_{1,10}=$ 11.27, $P=0.008$; SCPHT), but not in saline-treated rats ( $+1 \%$ versus saline-no stress, $F_{1,10}=0.003, P=1.91$; SCPHT; Fig. 4d). No changes were observed when analyzing the total levels of Pak1 (data not shown).

It has been postulated that long-term cocaine administration produces a reduction in the baseline neuronal activity of the $\mathrm{MPFC}$, but that exposure to a stimulus capable of generating drug-seeking (i.e. cocaine priming, cues or stress) would produce a hyperactive state (Jentsch \& Taylor 1999; Goldstein \& Volkow 2011). This may explain the higher responsivity to stress observed in the glutamate synapse of cocaine-treated rats. Accordingly, we decided to measure protein expression of the immediate early gene Arc, a well-established marker of neuronal activity. As shown in Fig. 5, two-way ANOVA indicated a significant cocaine $\times$ stress interaction $\left(F_{1,30}=12.54\right.$; $P=0.002)$. Examining the individual treatment effects, repeated exposure to cocaine reduced Arc expression $\left(-20 \%\right.$ versus saline-no stress, $F_{1,14}=5.90, P=0.035$; SCPHT); interestingly, Arc expression was significantly increased only in the mPFC of cocaine-withdrawn rats exposed to stress $(+39 \%$ versus cocaine-no stress, $F_{1,15}=20.96, P=0.0002$; SCPHT), but not in salinetreated rats $\left(-4 \%\right.$ versus saline-no stress, $F_{1,15}=0.16$, $P=0.692$; SCPHT; Fig. 5).

\section{DISCUSSION}

The present results show that adolescent cocaine exposure sensitizes glutamatergic responses of the $\mathrm{mPFC}$ to acute stress, presumably through cocaine-induced reduction of baseline mPFC neuronal activity that may generate a hyperactive state in response to a stimulus capable of generating drug seeking (i.e. acute stress; Jentsch \& Taylor 1999; Goldstein \& Volkow 2011). Our findings indicate that, in cocaine-withdrawn adolescent rats, the glutamate response to a brief stressor is dysregulated in the mPFC within a time frame of minutes. These findings may help to explain, at least in 
(a)

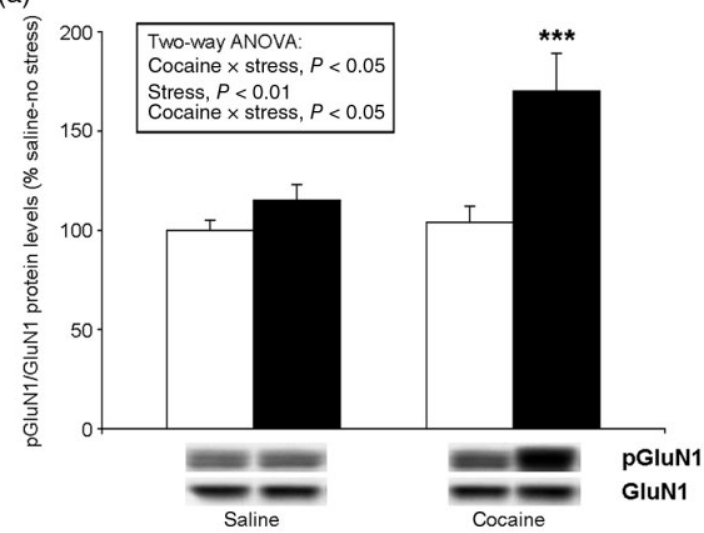

(c)

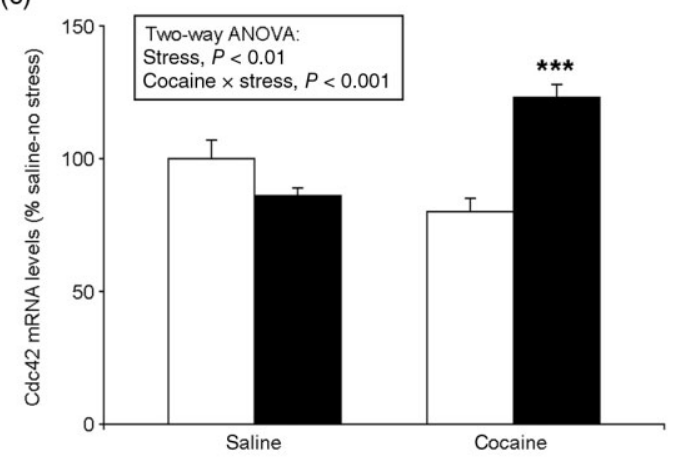

(b)

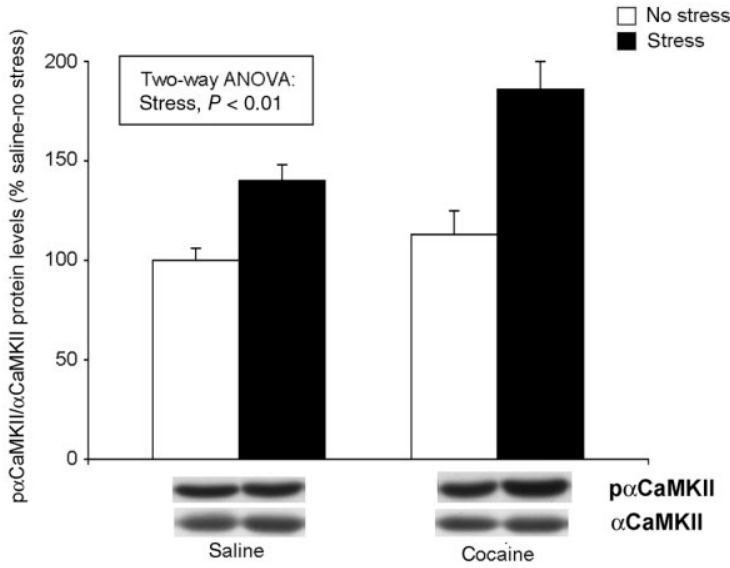

(d)

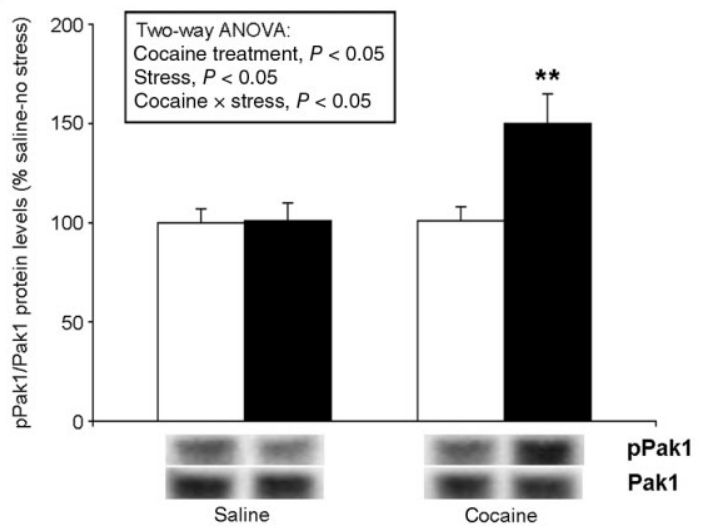

Figure 4 Effect of repeated cocaine treatment during adolescence (PND28 to PND 42) on postsynaptic determinants of glutamate transmission.

Panel (a) shows the activation of the NMDA GluNI receptor subunit whereas panel (b) shows the activation of the $\alpha$ CaMKII, both expressed as the ratio between the phosphorylated and the total form of the protein; panel (c) shows Cdc42 mRNA levels whereas panel (d) shows the phosphorylation of Pakl, expressed as the ratio between the phosphorylated and the total form of the protein. Asterisks denote the significant effect of the acute stress in cocaine-treated rats versus cocaine-no stress (** $P<0.0$ I; $* * * P<0.00$ I). Below the graphs in panel (a), (b) and (d) are shown representative bands of the respective proteins from Western blots

Table 1 Levels of glutamate NMDA receptor subunits GluN2A and GluN2B as well as of the AMPA receptor subunits GluA1 and GluA2 following the exposure to saline or cocaine during adolescence and subsequent exposure to stress in rat $\mathrm{mPFC}$.

\begin{tabular}{llccl}
\hline & Sal-sham & Sal-stress & Coc-sham & Coc-stress \\
\hline GluN2A & $100 \pm 14$ & $104 \pm 10$ & $110 \pm 9$ & $118 \pm 9$ \\
pGluN2B/GluN2B & $100 \pm 10$ & $95 \pm 15$ & $98 \pm 12$ & $112 \pm 11$ \\
pGluA1/GluA1 & $100 \pm 11$ & $152 \pm 13^{* *}$ & $101 \pm 9$ & $140 \pm 19^{* *}$ \\
pGluA2/GluA2 & $100 \pm 4$ & $100 \pm 6$ & $96 \pm 4$ & $107 \pm 7$ \\
\hline
\end{tabular}

The results represent the mean \pm standard error of measurement of at least six independent determinations for each experimental group $\left({ }^{* *} P<0.004\right)$. part, the hypersensitivity to stress observed in cocaine users during early abstinence (Sinha et al. 2003; Fox et al. 2008).

Effects of acute stress on glutamate-related glial elements in the mPFC of cocaine-treated rats

Glutamate signaling requires a dynamic and coordinated interplay among neurons and glial cells (Bridges et al.
2012). We found that the glial glutamate transporters EAAT1 and EAAT2 mRNA levels are reduced following the combination of cocaine and stress. This suggests that the clearance of the extracellular glutamate from the synaptic space is impaired after stress in the mPFC of cocaine-treated rats, pointing to increased excitatory neurotransmission in these animals. Interestingly, exposure to the combination of cocaine and stress also reduced the mRNA levels of the glutamate antiporter 


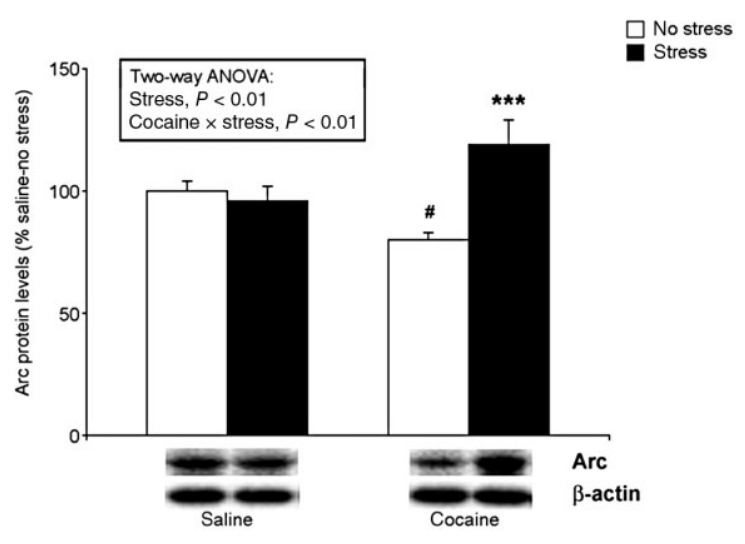

Figure 5 Effect of repeated cocaine treatment during adolescence (PND28 to PND 42) on Arc protein levels.

Dagger denotes the significant effect of repeated cocaine treatment during adolescence versus saline-no stress $\left({ }^{P} P<0.05\right)$. Asterisks denote the significant effect of the acute stress in cocaine-treated rats versus cocaine-no stress (**** $<0.00 \mathrm{I})$. Below the graph are shown representative bands of Arc protein from Western blots

system $X_{\mathrm{c}}^{-}$. Because this antiporter modulates the efflux of glutamate at extra-synaptic sites, which stimulates presynaptic mGluR 2/3 receptors to inhibit glutamate release (Baker et al. 2002), reduced expression of this antiporter by the combination of cocaine and stress may potentiate synaptic excitatory neurotransmission. The reduction of EAAT1 and EAAT2 mRNA levels may also be seen as an adaptive response to maintain physiological levels of glutamate in the extra-synaptic space to avoid excessive clearance.

We also observed a significant elevation of GS, the enzyme that converts glutamate into glutamine, in glial cells. Because glutamine is then released by glial cells and taken up by glutamate neurons (Broer \& Brookes 2001), increased expression of GS by the combination of cocaine and stress may lead to higher availability of glutamate inside the presynaptic glutamate neurons. Our data suggest that glial cells strongly contribute to the observed dysregulation of glutamate homeostasis in animals exposed to the combination of cocaine and stress through at least two different mechanisms, i.e. by reducing glutamate reuptake and by increasing glutamate availability at the presynaptic level.

Effects of acute stress on pre- and postsynaptic elements of the glutamatergic synapse in the mPFC of cocaine-treated rats

Interestingly, vGLUT1 mRNA levels were markedly increased in the mPFC of only rats exposed to the combination of cocaine and stress. Based on previously published evidence showing that vGLUT1 expression directly regulates glutamate release and the efficacy of glutamate neurotransmission (Wojcik et al. 2004; Wilson et al.
2005), our data suggest that more glutamate might be available for activity-dependent glutamate release, presumably as a consequence of the increased glutamine synthesis in glial cells mentioned earlier.

We then shifted our attention to the GABA network, as it has recently been shown that non-contingent cocaine exposure during adolescence disrupts GABA functions in the mPFC (Cass et al. 2013). In contrast to the mRNA levels of vGLUT1, the mRNA levels of vGAT, the protein responsible of GABA storage and release, were reduced in the $\mathrm{mPFC}$ of animals exposed to the combination of cocaine and stress. Because GABA is an inhibitory neurotransmitter, a reduction of vGAT may further contribute to the potentiation of the excitatory neurotransmission in cocaine-withdrawn rats exposed to stress. This is further strengthened by the evidence that the mRNA levels of GAD 67, the enzyme responsible of converting glutamate into GABA, is reduced in the $\mathrm{mPFC}$ of rats exposed to the combination of cocaine and stress suggesting a reduced conversion of glutamate into GABA and, therefore, an accumulation of the excitatory neutotransmitter.

The analysis of postsynaptic glutamatergic elements also revealed changes indicative of hyperresponsive glutamatergic synapse. In fact, we found a selective activation of the obligatory subunit GluN1 in the mPFC of rats exposed to the combination of cocaine and stress. Such activation of the NMDA receptor may increase calcium influx that, in turn, activates downstream kinases. To this end, we measured the phosphorylation of $\alpha$ CaMKII, a sensor of intracellular calcium levels (Coultrap \& Bayer 2012) and found it increased in the mPFC of cocaine-withdrawn rats. It is known that the activation of the NMDA receptor leads to changes in spine structural plasticity, an effect that may contribute to reinstatement of cocaine seeking (Toda et al. 2006), via the activation of the CaMKII-Cdc42-Pak signal transduction pathway (Murakoshi et al. 2011). Accordingly we measured both Cdc42 and Pak and found them enhanced by stress only in the mPFC of cocaine-treated rats. These data show increased responsiveness of cortical spines to stress only in animals with a previous history of cocaine exposure.

A potential limitation of our results may derive from the nature of cocaine exposure employed in the present study (i.e. non contingent) which is known to be more stressful relative to self-administration (Mantsch \& Goeders 2000); however, no differences in corticosterone levels were observed in the plasma of cocaine-treated rats when compared with saline-treated animals suggesting that the modality of cocaine treatment can not be considered a potential confound under our experimental conditions. Also, one may argue that we primarily rely on mRNA/phosphorylation measures to infer changes in 
(a)
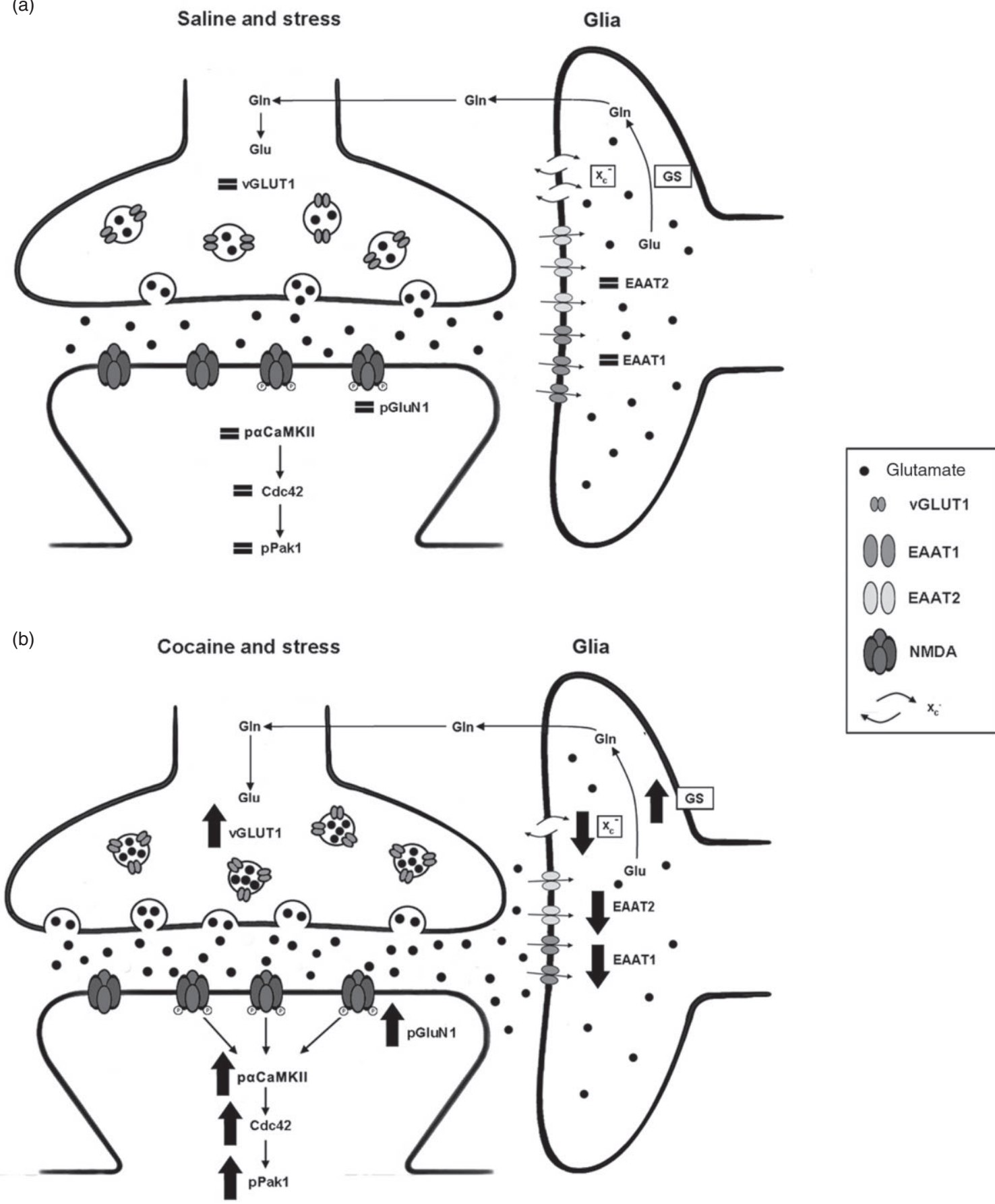

Figure 6 Exposure to repeated cocaine administration during adolescence alters the response of the cortical glutamate synapse to an acute stressor (panel b): comparison with rats exposed to saline and stress (panel a).

The homeostasis of the glutamate synapse is altered in the mPFC of rats exposed to the combination of cocaine and stress. In the cocaine + stress group, glial function is altered as evidenced by reduced expression of the main glial glutamate transporters EAATI and EAAT2 that leads to diminished clearance of extracellular glutamate. Changes in glial homeostasis are further revealed by a reduced expression of the cystine/glutamate antiporter, system $X_{c}^{-}$, which modulates non-vesicular glutamate release. Further, increased expression of GS leads to higher availability of glutamine that is released by astrocytes and taken up by presynaptic neurons. This may contribute to the increased expression of VGLUTI implying that more glutamate is available for activity-dependent glutamate release. The potentiation of the excitatory transmission results in increased activation of the postsynaptic NMDA receptor subunit GluNI and CaMKII and increased responsiveness of cortical spines as measured by increased Cdc42 expression and PakI phosphorylation. $\alpha \mathrm{CaMKII}=\alpha \mathrm{Ca}^{++} /{ }^{2}$ almodulin-dependent protein kinase; Cdc42, cell division cycle 42, GTP binding protein; EAATI, excitatory amino acid transporter I; EAAT2, excitatory amino acid transporter 2; Gln, glutamine; Glu, glutamate; GluNI, glutamate NMDA receptor subunit I; GS, glutamine synthetase; PakI, p2I-Activated Kinase I; vGLUTI, vesicular glutamate transporter $I ; X_{c}^{-}$, cystine/glutamate antiporter 
function. However, the evaluation of the rapid coping to the acute stress needs to rely on measuring changes in gene expression and/or phosphorylation as these represent the initial responses, whereas changes in corresponding protein levels take much longer.

\section{CONCLUSIONS}

Exposure to acute stress in adolescent cocaine-treated rats leads to widespread changes in both glial and synaptic regulators of glutamate neurotransmission leading to reduced glutamate clearance, increased packaging of glutamate in the presynaptic vesicular stores, increased conversion of glutamate to glutamine, all of which would be combined with data consistent with increased activation of NMDA receptors (Fig. 6). These results suggest that hyperreactive glutamatergic synapses in the mPFC may contribute to the hypersensitivity to stress observed in abstinent cocaine users (Sinha et al. 2003; Fox et al. 2008).

Furthermore, these hypersensitive glutamatergic synapses in the mPFC may contribute to the negative emotional state and stress-induced reinstatement generally observed in animal models of cocaine abuse (Baker et al. 2003; McFarland, Lapish \& Kalivas 2003; Koob 2008). Notably, when compared with saline-treated rats exposed to swim stress, cocaine-treated rats exposed to swim stress showed increased immobility, a measure of depressive-like behavior. The evidence that, in cocainetreated rats, acute stress recapitulates a depressive-like behavior that is usually only seen after chronic stress (Willner 1997; Kompagne et al. 2008) suggests that withdrawal from our cocaine paradigm may have led to a latent negative emotional state (Koob \& Le Moal 1997), which is precipitated by an acute stressor. Interestingly, most of the markers that were altered following the combination of cocaine and stress are, indeed, changed in animal models of depression (Banasr et al. 2010; Eriksson et al. 2012). The changes in glutamate homeostasis herein observed may therefore contribute, at least in part, to the pro-depressive symptoms occurring during the initial phase of cocaine withdrawal (Gawin 1991; Perrine et al. 2008).

\section{Acknowledgements}

We acknowledge TA Slotkin and KJ Valenzano for reading the article. This work was supported by Dipartimento delle Politiche Antidroga (DPA) (Rome, Italy), Grant 'CAINO 2' to FF.

\section{Disclosure/Conflict of Interest}

The authors report no biomedical financial interests or potential conflicts of interest.

\section{References}

Ahmed SH, Koob GF (2005) Transition to drug addiction: a negative reinforcement model based on an allostatic decrease in reward function. Psychopharmacology (Berl) 180:473490.

Baker DA, McFarland K, Lake RW, Shen H, Tang XC, Toda S, Kalivas PW (2003) Neuroadaptations in cystine-glutamate exchange underlie cocaine relapse. Nat Neurosci 6:743-749.

Baker DA, Xi ZX, Shen H, Swanson CJ, Kalivas PW (2002) The origin and neuronal function of in vivo nonsynaptic glutamate. J Neurosci 22:9134-9141.

Banasr M, Chowdhury GM, Terwilliger R, Newton SS, Duman RS, Behar KL, Sanacora G (2010) Glial pathology in an animal model of depression: reversal of stress-induced cellular, metabolic and behavioral deficits by the glutamate-modulating drug riluzole. Mol Psychiatry 15:501-511.

Barbon A, Fumagalli F, Caracciolo L, Madaschi L, Lesma E, Mora C, Carelli S, Slotkin TA, Racagni G, Di Giulio AM, Gorio A, Barlati S (2010) Acute spinal cord injury persistently reduces R/G RNA editing of AMPA receptors. J Neurochem 114:397407.

Bellocchio EE, Reimer RJ, Fremeau RT, Jr, Edwards RH (2000) Uptake of glutamate into synaptic vesicles by an inorganic phosphate transporter. Science 289:957-960.

Ben-Shahar O, Sacramento AD, Miller BW, Webb SM, Wroten MG, Silva HE, Caruana AL, Gordon EJ, Ploense KL, Ditzhazy J, Kippin TE, Szumlinski KK (2013) Deficits in ventromedial prefrontal cortex group 1 metabotropic glutamate receptor function mediate resistance to extinction during protracted withdrawal from an extensive history of cocaine selfadministration. J Neurosci 33:495-506.

Berridge KC, Robinson TE (1998) What is the role of dopamine in reward: hedonic impact, reward learning, or incentive salience? Brain Res Brain Res Rev 28:309-369.

Bridges R, Lutgen V, Lobner D, Baker DA (2012) Thinking outside the cleft to understand synaptic activity: contribution of the cystine-glutamate antiporter (System $X_{\mathrm{c}}^{-}$) to normal and pathological glutamatergic signaling. Pharmacol Rev 64:780-802.

Broer S, Brookes N (2001) Transfer of glutamine between astrocytes and neurons. J Neurochem 77:705-719.

Caffino L, Racagni G, Fumagalli F (2011) Stress and cocaine interact to modulate Arc/Arg3.1 expression in rat brain. Psychopharmacology (Berl) 218:241-248.

Cass DK, Thomases DR, Caballero A, Tseng KY (2013) Developmental disruption of gamma-aminobutyric acid function in the medial prefrontal cortex by noncontingent cocaine exposure during early adolescence. Biol Psychiatry. pii: S00063223(13)00189-3. doi: 10.1016/j.biopsych.2013.02.021. [Epub ahead of print]

Chapman RH, Stern JM (1978) Maternal stress and pituitaryadrenal manipulations during pregnancy in rats: effects on morphology and sexual behavior of male offspring. J Comp Physiol Psychol 92:1074-1083.

Collins SL, Izenwasser S (2004) Chronic nicotine differentially alters cocaine-induced locomotor activity in adolescent vs. adult male and female rats. Neuropharmacology 46:349362.

Coultrap SJ, Bayer KU (2012) CaMKII regulation in information processing and storage. Trends Neurosci 35:607-618.

Erb S, Shaham Y, Stewart J (1996) Stress reinstates cocaineseeking behavior after prolonged extinction and a drug-free period. Psychopharmacology (Berl) 128:408-412. 
Eriksson TM, Delagrange P, Spedding M, Popoli M, Mathe AA, Ogren SO, Svenningsson P (2012) Emotional memory impairments in a genetic rat model of depression: involvement of 5-HT/MEK/Arc signaling in restoration. Mol Psychiatry $17: 173-184$

Everitt BJ, Robbins TW (2005) Neural systems of reinforcement for drug addiction: from actions to habits to compulsion. Nat Neurosci 8:1481-1489.

Fox HC, Hong KI, Siedlarz K, Sinha R (2008) Enhanced sensitivity to stress and drug/alcohol craving in abstinent cocaine-dependent individuals compared to social drinkers. Neuropsychopharmacology 33:796-805.

Freeman WM, Patel KM, Brucklacher RM, Lull ME, Erwin M, Morgan D, Roberts DC, Vrana KE (2008) Persistent alterations in mesolimbic gene expression with abstinence from cocaine self-administration. Neuropsychopharmacology 33:18071817.

Fumagalli F, Caffino L, Racagni G, Riva MA (2009a) Repeated stress prevents cocaine-induced activation of BDNF signaling in rat prefrontal cortex. Eur Neuropsychopharmacol 19:402408.

Fumagalli F, Calabrese F, Luoni A, Bolis F, Racagni G, Riva MA (2012) Modulation of BDNF expression by repeated treatment with the novel antipsychotic lurasidone under basal condition and in response to acute stress. Int J Neuropsychopharmacol 15:235-246.

Fumagalli F, Pasini M, Frasca A, Drago F, Racagni G, Riva MA (2009b) Prenatal stress alters glutamatergic system responsiveness in adult rat prefrontal cortex. J Neurochem 109:1733-1744.

Gawin FH (1991) Cocaine addiction: psychology and neurophysiology. Science 251:1580-1586.

Ghasemzadeh MB, Mueller C, Vasudevan P (2009) Behavioral sensitization to cocaine is associated with increased glutamate receptor trafficking to the postsynaptic density after extended withdrawal period. Neuroscience 159:414-426.

Gipson CD, Kupchik YM, Kalivas PW (2013) Rapid, transient synaptic plasticity in addiction. Neuropharmacology. pii: S0028-3908(13)00178-0. doi: 10.1016/j.neuropharm .2013.04.032. [Epub ahead of print]

Goldstein RZ, Volkow ND (2011) Dysfunction of the prefrontal cortex in addiction: neuroimaging findings and clinical implications. Nat Rev Neurosci 12:652-669.

Hemby SE, Horman B, Tang W (2005) Differential regulation of ionotropic glutamate receptor subunits following cocaine selfadministration. Brain Res 1064:75-82.

Huang CC, Yeh CM, Wu MY, Chang AY, Chan JY, Chan SH, Hsu KS (2011) Cocaine withdrawal impairs metabotropic glutamate receptor-dependent long-term depression in the nucleus accumbens. J Neurosci 31:4194-4203.

Jentsch JD, Taylor JR (1999) Impulsivity resulting from frontostriatal dysfunction in drug abuse: implications for the control of behavior by reward-related stimuli. Psychopharmacology (Berl) 146:373-390.

Kalivas PW, Volkow N, Seamans J (2005) Unmanageable motivation in addiction: a pathology in prefrontal-accumbens glutamate transmission. Neuron 45:647-650.

Kasanetz F, Lafourcade M, Deroche-Gamonet V, Revest JM, Berson N, Balado E, Fiancette JF, Renault P, Piazza PV, Manzoni OJ (2013) Prefrontal synaptic markers of cocaine addiction-like behavior in rats. Mol Psychiatry 18:729737.

Kompagne H, Bardos G, Szenasi G, Gacsalyi I, Harsing LG, Levay G (2008) Chronic mild stress generates clear depressive but ambiguous anxiety-like behaviour in rats. Behav Brain Res 193:311-314

Koob GF (2008) A role for brain stress systems in addiction. Neuron 59:11-34

Koob GF, Le Moal M (1997) Drug abuse: hedonic homeostatic dysregulation. Science 278:52-58.

Mantsch JR, Goeders NE (2000) Effects of cocaine selfadministration on plasma corticosterone in rats: relationship to hippocampal type II glucocorticoid receptors. Prog Neuropsychopharmacol Biol Psychiatry 24:633-646.

McFarland K, Lapish CC, Kalivas PW (2003) Prefrontal glutamate release into the core of the nucleus accumbens mediates cocaine-induced reinstatement of drug-seeking behavior. J Neurosci 23:3531-3537.

Murakoshi H, Wang H, Yasuda R (2011) Local, persistent activation of Rho GTPases during plasticity of single dendritic spines. Nature 472:100-104.

Paxinos G, Watson C (2005) The Rat Brain in Stereotaxic Coordinates, 5th edn. New York: Academic Press.

Perrine SA, Sheikh IS, Nwaneshiudu CA, Schroeder JA, Unterwald EM (2008) Withdrawal from chronic administration of cocaine decreases delta opioid receptor signaling and increases anxiety- and depression-like behaviors in the rat. Neuropharmacology 54:355-364.

Purgianto A, Scheyer A, Loweth JA, Ford KA, Tseng KY, Wolf ME (2013) Different adaptations in AMPA receptor transmission in the nucleus accumbens after short versus long access cocaine self-administration regimens. Neuropsychopharmacology 38:1789-1797.

Sanacora G, Treccani G, Popoli M (2012) Towards a glutamate hypothesis of depression: an emerging frontier of neuropsychopharmacology for mood disorders. Neuropharmacology 62:63-77.

Sinha R, Talih M, Malison R, Cooney N, Anderson GM, Kreek MJ (2003) Hypothalamic-pituitary-adrenal axis and sympathoadreno-medullary responses during stress-induced and drug cue-induced cocaine craving states. Psychopharmacology (Berl) 170:62-72.

Soghomonian JJ, Martin DL (1998) Two isoforms of glutamate decarboxylase: why? Trends Pharmacol Sci 19:500-505.

Toda S, Shen HW, Peters J, Cagle S, Kalivas PW (2006) Cocaine increases actin cycling: effects in the reinstatement model of drug seeking. J Neurosci 26:1579-1587.

Willner P (1997) Validity, reliability and utility of the chronic mild stress model of depression: a 10-year review and evaluation. Psychopharmacology (Berl) 134:319-329.

Wilson NR, Kang J, Hueske EV, Leung T, Varoqui H, Murnick JG, Erickson JD, Liu G (2005) Presynaptic regulation of quantal size by the vesicular glutamate transporter VGLUT1. J Neurosci 25:6221-6234.

Wojcik SM, Rhee JS, Herzog E, Sigler A, Jahn R, Takamori S, Brose N, Rosenmund C (2004) An essential role for vesicular glutamate transporter 1 (VGLUT1) in postnatal development and control of quantal size. Proc Natl Acad Sci U S A 101:7158-7163.

Wong WC, Ford KA, Pagels NE, McCutcheon JE, Marinelli M (2013) Adolescents are more vulnerable to cocaine addiction: behavioral and electrophysiological evidence. J Neurosci 33:4913-4922.

Xie X, Lasseter HC, Ramirez DR, Ponds KL, Wells AM, Fuchs RA (2012) Subregion-specific role of glutamate receptors in the nucleus accumbens on drug context-induced reinstatement of cocaine-seeking behavior in rats. Addict Biol 17:287299. 